\title{
BMJ Open Prevalence and risk factors for food allergy in older people: protocol for a systematic review
}

\author{
Inês Laia-Dias, ${ }^{1}$ Carlos Lozoya-lbáñez, ${ }^{2}$ Isabel Skypala, ${ }^{3}$ Jorge M R Gama, ${ }^{4}$ \\ Ulugbek Nurmatov, ${ }^{5}$ Olga Lourenço, ${ }^{1,6}$ Luís Taborda-Barata ${ }^{6,7}$
}

To cite: Laia-Dias I, Lozoya-Ibáñez C, Skypala I, et al. Prevalence and risk factors for food allergy in older people: protocol for a systematic review. BMJ Open 2019;9:e029633. doi:10.1136/ bmjopen-2019-029633

- Prepublication history and additional material for this paper are available online. To view these files, please visit the journal online (http://dx.doi. org/10.1136/bmjopen-2019029633).

IL-D and CL-I are joint first authors.

Received 02 February 2019

Revised 09 July 2019

Accepted 10 July 2019

Check for updates

(C) Author(s) (or their employer(s)) 2019. Re-use permitted under CC BY-NC. No commercial re-use. See rights and permissions. Published by BMJ.

For numbered affiliations see end of article.

Correspondence to

Professor Luís Taborda-Barata; tabordabarata@fcsaude.ubi.pt

\section{ABSTRACT}

Introduction Studies suggest that the prevalence of food allergy may be increasing worldwide. Results regarding the prevalence and features of adverse food reactions older people have, however, scarcely been analysed in the literature. Thus, the objective of the present systematic review will be to describe the prevalence of food allergy in older individuals, its risk factors, clinical features, as well as the most frequently and commonly involved foods. Methods and analysis We will conduct a systematic review and meta-analysis of the incidence, prevalence and risk factors for food allergy in older individuals. We will search international electronic databases including MEDLINE, EMBASE, Cochrane Library, CINAHL, AMED and ISI Web of Science for published, unpublished and ongoing studies from 1980 toJanuary 2019. There will be no restriction on the language or geography of publication. We will use the critical appraisal skills programme quality assessment tool to appraise the methodological quality of included studies. A descriptive summary with data tables will be elaborated, and if deemed clinically relevant and statistically adequate, meta-analysis using random-effects modelling will be carried out, given the expected clinical, methodological and statistical heterogeneity of studies. The Preferred Reporting Items for Systematic Reviews and Meta-Analyses checklist will guide reporting of the systematic review.

Ethics and dissemination Since this systematic review will be solely based on published and retrievable literature, no ethics approval will be obtained. This study will allow us to draw up-to-date estimates of the prevalence of adverse food reactions in older individuals, worldwide, besides allowing the identification of its major risk factors, clinical manifestations and predominant foods responsible for such reactions. A multidisciplinary team has been assembled for this systematic review and will participate in relevant dissemination activities, namely reports, publications and presentations.

PROSPERO registration number CRD42018102140

\section{BACKGROUND}

The prevalence of food allergies in the general adult population is less well known than in children, since there are fewer studies in the former. Nevertheless, meta-analyses have estimated the prevalence of food allergy in adults to vary between $3.5 \%$ and $35 \%$ when

\section{Strengths and limitations of this study}

Food allergy is a growing problem worldwide namely in older individuals.

- This is the first systematic review which will specifically address issues related to food allergy in older people, which may have clinical implications.

- A thorough and highly sensitive search strategy in leading databases, with no geographical or language restrictions, will be conducted by a multidisciplinary team with expertise in the field.

- Study heterogeneity in terms of operational definitions of food allergy may hinder a meta-analysis.

only based on self-report, and between $2 \%$ and $4 \%$ when studies include more stringent additional criteria such as positive skin prick tests (SPT) and/or food-specific IgE levels or the gold standard of double-blind placebo-controlled food challenge. ${ }^{1-3}$ In addition, the prevalence of food allergy may be increasing worldwide, not only in western countries but also in other countries which have adopted a westernised living style. ${ }^{14}$

However, it should be borne in mind that epidemiological studies of food allergies most frequently focus on children and young adults, and reports that specifically include older individuals are scarce. ${ }^{1-35}$ In fact, most epidemiological results of food allergy involving older people are included in studies that addressed this issue in global populations of adults. Overall, it is not clear whether the prevalence of food allergy is similar, lower or higher in older individuals than in young adults or in children. In this context, a previous meta-analysis has shown that it may be higher in older Europeans, ${ }^{1}$ although a second, previous meta-analysis, which screened studies from European and non-European countries showed that the prevalence of food allergy was lower in adults than in children ${ }^{2}$; however, the latter study only used aggregated data, and did not 
specifically analyse older adults. Thus, further studies are necessary to clarify this issue. Nevertheless, the prevalence of food allergy may also be increasing in older individuals. For example, the analysis of the US Food and Drug Administration Food Safety Surveys study, which are cross-sectional, telephone surveys of adult American consumers conducted every 3-5 years since 1988 showed that the prevalence of self-reported food allergy increased between 2001 and 2010 in older individuals, although this was only significant in the 60-year-old to 69-year-old group (an increase from $7.7 \%$ to $11.7 \%$; $\mathrm{p}<0.002$ ), but not in the $>70$-year-old group (increase from $8.7 \%$ to $10.6 \%$ but $\mathrm{p}=0.337){ }^{6}$

It should also be taken into account that the numbers and relative percentage of older people are increasing worldwide. According to the United Nations, ${ }^{7}$ in 2017 , $13 \%$ of the world population was aged 60 years or over and $2 \%$ was aged 80 years or over. In comparison with 2017 , by 2050 , the population aged 60 years and over is expected to increase twofold (962 million to 2.1 billion), and the population aged 80 years and over may threefold (137 million to 425 million).

The ageing process is accompanied by immunophysiological and biochemical changes that may make food allergies manifest differently in older people, a situation which may be further compounded by concurrent medications and comorbidities, as well as lack of awareness of the problem. ${ }^{589}$ These factors may lead to underdiagnosis and undertreatment of food allergies in older individuals. ${ }^{58}$ Furthermore, these changes might be reflected not only in clinical manifestations of food allergy but also in positivity of skin test results or levels of food-specific IgE antibodies, which may result in differences in detectable prevalence and risk factors, as well as in predominant foods associated with food allergy in older people. All of these points may demand a different approach regarding its diagnosis and management in comparison with younger adults. ${ }^{5}$ However, to the best of our knowledge, no previous systematic review has been published on epidemiological aspects of food allergies specifically in older individuals.

Thus, the objectives of this systematic review will be: (1) to describe the worldwide prevalence, and time trends of food allergy in older people, (2) to describe clinical manifestations and predominant foods associated with food allergy in older people; (3) to analyse risk and prognostic factors associated with food allergy in older individuals.

\section{METHODS AND ANALYSIS \\ Search strategy}

The summary of this systematic review protocol has been registered in the International Prospective Register of Systematic Reviews (PROSPERO) ${ }^{10}$.

We have developed a comprehensive search strategy for screening published and unpublished studies. As sources of published studies, we will search the Cochrane Library (Cochrane Database of Systematic Reviews, Cochrane
Central Register of Controlled Trials, Cochrane Methodology Register), MEDLINE, EMBASE, CINAHL, AMED and ISI Web of Science (Science and Social Science Index).

The bibliographies of all eligible studies will also be scrutinised to identify additional possible studies. Unpublished and research in progress will be searched in key internet-based relevant databases-www.clinicaltrials.gov; http://www.isrctn.com/ (ISRCTN Registry); www.anzctr. org.au. In addition, to extend our search for published, unpublished and ongoing studies, we will contact an international panel of experts in this field.

Studies from all over the world will be included, if they meet the inclusion/exclusion criteria. No language restrictions will be imposed; translations will be undertaken where necessary. We will report any literature that we are unable to translate. Search dates will be from 1980 untilJanuary 2019. Search terms are detailed in online supplementary appendix 1 . If any changes are made to the protocol, these will be registered by submission of an updated version to PROSPERO, and will also be documented on the final manuscript with the results of the systematic review.

\section{Inclusion criteria for study designs}

We will include all observational, including cohort, case-control and cross-sectional studies. In addition, systematic reviews and meta-analyses with the same focus will be scrutinised. These study designs were selected to ensure the selection and pooling of the highest possible level of evidence based on the aims of this review.

In terms of population, we will select studies that include (not only exclusively) participants aged 60 years or older, reporting or having a diagnosis of food allergy. This cut-off age will used as a criterion for considering an individual as 'older adult' since our systematic review will include studies from all over the world, and the WHO proposed 60 years as a working definition of an 'older person' in African countries. ${ }^{11}$ In addition, although 65 years is recommended by WHO as a cut-off level in western countries, ${ }^{12} 13$ and this is the threshold used in most studies in older individuals in those countries, there are some epidemiological studies also performed in such countries which use 60-year cut-off age for identifying older people. ${ }^{6}$ Thus, we will include data from all individuals who are 60 years or older, in order to ensure that our study will be fully inclusive.

The following study designs will be excluded: narrative literature reviews, discussion papers, non-research letters and editorials, case studies and case series, animal studies.

\section{Study selection}

Titles and abstracts of included papers will be independently checked by two investigators. The full text of all potentially eligible studies will be retrieved and independently assessed against the inclusion criteria (see above) by two reviewers. The reviewers will decide which of the studies fit the inclusion criteria: any disagreements 
will be resolved by discussion, with a third researcher brought in to arbitrate if needed.

To ensure transparency, the process of selection will be summarised using a Preferred Reporting Items for Systematic Reviews and Meta-Analyses flow diagram.

\section{Data extraction}

Data from selected articles will be extracted independently by two reviewers who will transfer data from their original presentation to a proper form made in Microsoft Excel software, with each study receiving a reference code. Any discrepancy will be resolved by discussion with the third reviewer. If an article presents results from $\mathrm{N}$ different studies, then, $\mathrm{N}$ different forms will be created to collect data. Before using the form, we will test it in a pilot extraction step with a selected sample of studies. This will allow us to check the capacity of the constructed for to capture the relevant information that will be used for analysis.

If necessary, we will collect indirect data from figures and charts, adapting their interpretation from two different authors by consensus, and authors of original articles will also be contacted for further information and data. In articles in which data from older patients were analysed together with those from younger patients, authors will be contacted in order to clarify or make available data pertaining to the former group, for subgroup analyses.

\section{Data items}

The following information will be collected from selected studies involving older individuals, using the same approach that was previously used in a systematic review protocol which involved all epidemiological parameters of food allergies in European individuals of various ages but which did not focus on older individuals ${ }^{14}$ : (1) frequency of food allergy (i) by self-report; (ii) by clinical symptoms plus positive SPT or IgE to food allergens; (iii) by clinical symptoms, positive SPT or IgE to food allergens and also food challenge confirmed; (2) most frequently involved food allergens; (3) most frequently observed symptoms and symptom clusters; (4) timeframe of symptom development on ingestion of foods; (5) time trends in frequency of food allergy; (6) geographical differences in prevalence of food allergy and related food allergens; (7) risk factors for food allergy.

\section{Outcome assessment}

Diverse methods of assessment have been used to define food allergy in different studies. Thus, for estimation of the prevalence (point, period and lifetime prevalence) and incidence (incidence rate, cumulative incidence) of food allergies, we will include all methods that were used in previous primary studies, including self-reported assessment, clinician diagnosis, allergic sensitisation (based on SPT results, skin prick-prick test results, food allergen-specific IgE levels, skin atopy patch tests) and food challenges (open, single-blinded, double-blinded).
However, analyses will take into account each such type of operational definition of food allergy in epidemiological studies.

Regarding the analysis of risk factors and clinical manifestations of adverse food reactions, we will only include studies that have studied objectively confirmed food allergic reactions (using food challenges), since this will ensure the most robust approach to assessing a potential causal relationship between the studied risk factors and the studied outcome (food allergy as expressed by food-induced symptoms in a food challenge). This approach was also followed by the previously mentioned systematic review by Nwaru et al, which studied the epidemiology of food allergy for all ages, in Europe. ${ }^{1}$

\section{Risk of bias assessment strategy}

Risk of bias assessment will be independently verified by two different reviewers for each individual study that will be selected, using the critical appraisal skills programme quality assessment tool for the types of included studies, including assessment of internal and external validity. ${ }^{15-17}$ We will assess heterogeneity, consistency and risk of bias. Quality of evidence and recommendation for the different outcomes will be assessed using the GRADE (Grading of Recommendations Assessment, Development and Evaluation) system. ${ }^{18}$

All studies and their individual elements will be graded in terms of adequacy of the study regarding the research question, risk of selection bias, measurement of exposure and assessment of outcomes. Disagreements will be resolved by a third reviewer.

\section{Analysis, data synthesis, publication bias and reporting}

A narrative synthesis of the data will be performed. In addition, a descriptive summary with data tables will be elaborated, in order to summarise literature findings, ${ }^{19}$ and if deemed clinically relevant and statistically adequate, meta-analysis using random-effects modelling will be carried out. ${ }^{20-22}$ Forest plot and Funnel plot charts will be made, if necessary, to compare results or to identify publication bias, since publication bias leads to funnel plot asymmetry, if 10 or more relevant studies are detected. ${ }^{23}$ Begs and Egger's methods will be used for testing such funnel plot asymmetry. ${ }^{24}{ }^{25}$ Heterogeneity between studies will be analysed using the the $\mathrm{I}^{2}$ statistical index.$^{26}$ Subgroup analysis may eventually be carried out using the following age groups: 60-65, 66-80 and $>80$ years, if appropriate and if such data can be retrieved from the literature of after contacting authors. Statistical analysis will be carried out using SPSS V.25.0. Finally, the Preferred Reporting Items for Systematic Review and Meta-Analysis Protocols statement and checklist will be followed for reporting of the systematic review. ${ }^{27} 28$

\section{Ethics, dissemination data protection}

Ethical approval was not obtained since the data to be collected and analysed cannot be linked to specific individuals. A data management plan will be implemented in 
cases in which data from specific studies can be accessed directly or obtained from article authors. Retrieved data will be kept in a database that will have protected access and will only be used by the involved authors.

\section{Patient and public involvement}

Since this will be a systematic review, there will be no direct patient or public involvement.

\section{Ethics and dissemination}

This systematic review, based on studies published between 1980 and January 2019, will allow us to make assessments and estimates considering the appropriateness of the study design regarding the questions, methods used and risk of selection bias.

More specifically, one strength of the review is that it is novel in that we will provide estimates on the following aspects of food allergy with a focus on older individuals: (1) worldwide prevalence of food allergy in this subgroup of adults; (2) geographical differences in prevalence of food allergy and related food allergens; (3) time trends in prevalence of food allergy and related food allergens; (4) predominant foods associated with food allergy; (5) most frequent symptoms/ symptom clusters, as well as their severity, associated with food allergy; (6) most frequent symptoms associated with specific foods; (7) timeframe of symptom development on ingestion of foods; (8) need for treatment of episodes of food allergy; (9) risk factors associated with food allergy; (10) quality of life due to food allergy (if enough data are available).

Our results will potentially allow drawing conclusions about general and specific aspects of food allergies in older people. This information may be crucial to analysing similarities and differences regarding food allergies between older and younger individuals and eventually defining preventive or diagnostic approaches specifically tailored to the former age group.

Our dissemination strategy will involve presentation at scientific meetings, as well as publication of article(s) in international, peer-reviewed, open-access journals. However, given the increasing relative percentage of older people in the population, the relative lack of awareness of food allergy in this age group, as well as the inherent difficulties in diagnosing food allergies in older individuals, we also plan to organise meetings with general practitioners and other healthcare providers, to analyse and discuss our findings and their potential implications.

\section{Author affiliations}

${ }^{1}$ Faculty of Health Sciences, Universidade da Beira Interior, Covilhã, Portugal ${ }^{2}$ Department of Allergy and Clinical Immunology, Hospital Amato Lusitano, Castelo Branco Local Health Unit, Castelo Branco, Portugal

${ }^{3}$ Royal Brompton Hospital, Royal Brompton \& Harefield NHS Trust, London, UK ${ }^{4}$ Centre of Mathematics and Applications, Faculty of Sciences, Universidade da Beira Interior, Covilhã, Portugal

${ }^{5}$ Division of Population Medicine, School of Medicine, Cardiff University, Wales, UK ${ }^{6} \mathrm{CICS}$ - Health Sciences Research Centre, NuESA - Environment and Health Study Group, Faculty of Health Sciences, University of Beira Interior, Covilhã, Portugal ${ }^{7}$ Department of Allergy and Clinical Immunology, Cova da Beira University Hospital Centre, Covilhã, Portugal
Acknowledgements The authors would like to acknowledge Dr Rosa Saraiva, main librarian at the Cova da Beira University Hospital Centre, and Head of the Research and Innovation Department of this institution, for invaluable input in terms of discussion of this manuscript. In addition, the authors would also like to thank $\mathrm{Dr}$ Bright Nwaru, Group Leader at the Institute of Medicine, University of Gothenburg, Sweden, for his precious comments regarding the initial steps of designing the search strategy.

Contributors IL-D and CL-I are equal contributors to the design and conceptualisation of this review, and drafted the protocol with primary support from UN (review guarantor) and LT-B. UN, IS and OL were involved in checking various steps of the search strategy, including keywords, as well as the final version of the protocol. JMRG was involved in the statistical strategy for data analysis. IL-D, CL-I and LT-B were involved in establishing eligibility criteria and data extraction forms. All authors provided feedback on the manuscript, at all stages.

Funding The authors have not declared a specific grant for this research from any funding agency in the public, commercial or not-for-profit sectors.

Competing interests None declared.

Patient consent for publication Not required.

Provenance and peer review Not commissioned; externally peer reviewed.

Open access This is an open access article distributed in accordance with the Creative Commons Attribution Non Commercial (CC BY-NC 4.0) license, which permits others to distribute, remix, adapt, build upon this work non-commercially, and license their derivative works on different terms, provided the original work is properly cited, appropriate credit is given, any changes made indicated, and the use is non-commercial. See: http://creativecommons.org/licenses/by-nc/4.0/.

\section{REFERENCES}

1. Nwaru BI, Hickstein L, Panesar SS, et al. The epidemiology of food allergy in Europe: a systematic review and meta-analysis. Allergy 2014;69:62-75

2. Rona RJ, Keil T, Summers C, et al. The prevalence of food allergy: a meta-analysis. J Allergy Clin Immunol 2007;120:638-46.

3. Chafen JJ, Newberry SJ, Riedl MA, et al. Diagnosing and managing common food allergies: a systematic review. JAMA 2010;303:1848-56

4. Tang MLK, Mullins RJ. Food allergy: is prevalence increasing? Intern Med J 2017;47:256-61.

5. Jensem-Jarolim E, Jensen SAF. Food allergies in the elderly. Collecting the evidence. Ann Allergy Asthma Immunol 2016;117:472-5.

6. Verrill L, Bruns R, Luccioli S. Prevalence of self-reported food allergy in U.S. adults: 2001, 2006, and 2010. Allergy Asthma Proc 2015;36:458-67.

7. United Nations,, Department of Economic and Social Affairs,, Population Division. World population prospects: the 2017 revision, key findings and advance tables. Working paper No. ESA/P/WP/ 2017;248.

8. Diesner SC, Untersmayr E, Pietschmann P, et al. Food allergy: only a pediatric disease? Gerontology 2011;57:28-32.

9. Montanaro A. Allergic disease management in the elderly: a wakeup call for the allergy community. Ann Allergy Asthma Immunol 2000;85:85-6.

10. Laia-Dias I, Lozoya-Ibáñez C, Skypala I, et al. Prevalence and risk factors for food allergy in elderly individuals: protocol for a systematic review. Prospero 2018: CRD42018102140. Available: http://www.crd.york.ac.uk/PROSPERO/display_record.php?ID= CRD42018102140

11. W.H.O. Health Statistics and Information Systems.. Proposed working definition of an older person in Africa for the MDS project. Available: https://www.who.int/healthinfo/survey/ageingdefnolder/en/ [Accessed 7th Jan 2019].

12. World Health Organisation. Definition of an older or elderly person. Geneva: Switzerland: WHO, 2010. http://www.who.int/healthinfo/ survey/ageingdefnolder/en/index.html

13. Orimo $\mathrm{H}$, Ito $\mathrm{H}$, Suzuki $\mathrm{T}$, et al. Reviewing the definition of "elderly". Geriatr Gerontol Int 2006;6:149-58.

14. Nwaru BI, Panesar SS, Hickstein L, et al. The epidemiology of food allergy in Europe: protocol for a systematic review. Clin Transl Allergy 2013:3:13.

15. CASP checklist for systematic reviews. Available: https://www.caspuk.net/wp-content/ uploads/2018/03/CASP-Systematic-ReviewChecklist-2018_fillable-form.pdf [Accessed 22nd Dec 2018]. 
16. CASP checklist for cohort studies. Available: https://www.casp-uk. net/wp-content/ uploads/2018/03/CASP-Cohort-Checklist-2018_ fillable_form.pdf [Accessed 22nd Dec 2018].

17. CASP checklist for case-control studies. Available: https://www. casp-uk.net/wp-content/ uploads/2018/03/CASP-Case-ControlChecklist-2018_fillable_form.pdf [Accessed 22nd Dec 2018].

18. Balshem $\mathrm{H}$, Helfand $\mathrm{M}$, Schünemann $\mathrm{HJ}$, et al. Grade guidelines: 3 . rating the quality of evidence. J Clin Epidemiol 2011;64:401-6.

19. Guyatt G, Oxman AD, Akl EA, et al. Grade guidelines: 1. IntroductionGRADE evidence profiles and summary of findings tables. J Clin Epidemiol 2011;64:383-94.

20. Agresti A, Coull BA. Approximate is better than "exact" for interval estimation of binomial proportions. Am Statis 1998;52:119-26.

21. Borenstein M, Hedges LV, Higgins JPT, et al. A basic introduction to fixed-effect and random-effects models for meta-analysis. Res Synth Methods 2010;1:97-111.
22. Rice K, Higgins JPT, Lumley T. A re-evaluation of fixed effect(s) metaanalysis. J R Stat Soc Ser A Stat Soc 2018;181:205-27.

23. Sterne JAC, Harbord RM. Funnel plots in meta-analysis. Stata $J$ 2004:4:127-41.

24. Begg CB, Mazumdar M. Operating characteristics of a RANK correlation test for publication bias. Biometrics 1994;50:1088-101.

25. Egger M, Smith GD, Schneider M, et al. Bias in meta-analysis detected by a simple, graphical test. BMJ 1997;315:629-34.

26. Higgins JPTet al. Measuring inconsistency in meta-analyses. BMJ 2003;327:557-60.

27. Liberati A, Altman DG, Tetzlaff J, et al. The PRISMA statement for reporting systematic reviews and meta-analyses of studies that evaluate health care interventions: explanation and elaboration. PLoS Med 2009;6:e1000100.

28. Shamseer L, Moher D, Clarke M, et al. Preferred reporting items for systematic review and meta-analysis protocols (PRISMA-P) 2015 elaboration and explanation. BMJ 2015;349:97647. 